\title{
Synthesis and Crystal Structure of
}

\section{3-3'-Diaminomethyldipropylammonium Hexachlorobismuthate (III)}

\section{Yosra baklouti ${ }^{\mathrm{a} *}$, Abdelaziz Koumina ${ }^{\mathrm{b}}$, Mohamed Fliyou ${ }^{\mathrm{b}}$, Fatma zouari ${ }^{\mathrm{a}}$}

${ }^{a}$ Laboratoire des Sciences des Matériaux et d'Environnement, Faculté des Sciences de Sfax, BP 1171, 3000, Sfax, Tunisia

E-mail address: bakloutiyosra@ yahoo.fr; fatmazouari2003@yahoo.fr

${ }^{\mathrm{b}}$ ENS de Marrakech, Département de physique, Université Cadi-Ayyad Marrakech, Morocco.

E-mail address: koumina@gmail.com; fliyou@hotmail.com

\section{Abstract}

Synthesis and crystal structure of 3-3'-diamino-N-methyldipropylammonuim hexachlorobismuthate (III) are reported. The compound crystallizes in the triclinic system with space group P1. The unit cell dimensions are: $\mathrm{a}=7.5580(5), \mathrm{b}=7.8710(6), \mathrm{c}=8.3709(7) \AA$ with $\mathrm{Z}=1$. The crystal is built up of separated $\left[\mathrm{BiCl}_{6}\right]^{3-}$ octahedral anions and 3-3'-diamino- $\mathrm{N}$ - methyldipropylammonium cations. The organic layers are arranged in sandwich between the anionic ones. The crystal packing is governed by means of the ionic $\mathrm{N}-\mathrm{H} \cdots \mathrm{Cl}$ hydrogen bonds, forming a three dimensional network.

\section{Keywords:}

Organic-inorganic hybrid material; Crystal structure; Halogenobismuthates(III).

*Corresponding author: Yosra Baklouti.

Tel: +21624916258, fax +21674274437

E-mail address:bakloutiyosra@yahoo.fr

Address: Route de Soukra km 3.5 - B.P. n 1171 - 3000 Sfax.

\section{Council for Innovative Research}

Peer Review Research Publishing System

Journal: Journal of Advances in Chemistry

Vol. 8, No. 1

editor@cirworld.com

www.cirworld.com, member.cirworld.com 


\section{Introduction}

Recently, much attention has been focused on organic-inorganic hybrid materials based on metal-halide units; those materials are frequently characterized by their various physical and chemical properties that could lead to technological innovations like magnetic or ferroelectric transitions, conductivity (super conductivity), electroluminescence and photoluminescence [1-6]. To date, numerous metal-halide systems involving $\mathrm{Sn}, \mathrm{Pb}, \mathrm{Sb}, \mathrm{Bi}$, and $\mathrm{Te}$ have been synthesized and structurally characterized [7-12]. The anionic metal-halide species has been observed to range in dimensionality from two-dimensional or one dimensional polymeric anions to discrete anions of various sizes. The polyhedral may be linked by corner-, edge-, or face-sharing into numerous different arrangements; and consequently, halometallate compounds exhibit very great structural diversity $[8,10,13]$. The identity of the anions formed has been found to depend on the specific reaction conditions employed including the size, shape, the reagent stoichiometry, and ability to form the hydrogen bond system by the cations. The bismuth compounds represent a potential class of materials with unusual structural arche-types, due to the fact that the $\mathrm{Bi}(\mathrm{III})$ ion exhibits a variety of coordination modes, depending on crystal packing and on ligands: octahedral coordination being observed.

The polyhedra may be linked by corner-, edge-, or face sharing into numerous different arrangements, leading to an extensive family of bismuth(III) halogenoanions ([BiX $]^{-},\left[\mathrm{BiX}_{5}\right]^{2-},\left[\mathrm{BiX}_{6}\right]^{3-},\left[\mathrm{Bi}_{2} \mathrm{X}_{9}\right]^{3-},\left[\mathrm{Bi}_{2} \mathrm{X}_{11}\right]^{5-},\left[\mathrm{Bi}_{3} \mathrm{X}^{12}\right]^{3-},\left[\mathrm{Bi}_{4} \mathrm{X}_{18}\right]^{6-}$, $\left[\mathrm{Bi}_{6} \mathrm{X}_{22}\right]^{4-}$, and $\left.\left[\mathrm{Bi}_{8} \mathrm{X}_{30}\right]^{4-}\right)$ [14-19]. The structure of the anionic form is related to the size and symmetry of the organic counter-ions and their ability to form hydrogen bonds as well. In fact, besides the rich structural diversity displayed by these systems, some interest has been directed towards halobismuthate(III) compounds in combination with organic cations, due to their very interesting physical properties caused by active lone pairs [20].

In an attempt to study the effects of the size and the coordination mode of the cation in this class of compounds, we have successfully synthesized a new compound of formula $\left[\mathrm{C}_{7} \mathrm{H}_{22} \mathrm{~N}_{3}\right] \mathrm{BiCl}_{6}$.

In the present paper, we report the synthesis and the structural characterization by X-ray diffraction of the 3-3'-diamino- $\mathrm{N}$ methyldipropylammonium hexachlorobismuthate (III).

\section{Experimental section}

\section{II.1.Synthesis}

Single crystals of the title compound were prepared at $300 \mathrm{~K}$ by slow evaporation of a saturated aqueous solution obtained by dissolving $\mathrm{C}_{7} \mathrm{H}_{19} \mathrm{~N}_{3}$ and $\mathrm{BiCl}_{3}$ (molar ratio $3 / 1$ ) in $36 \% \mathrm{HCl}$. The resulting solution is kept under ambient conditions which were allowed to evaporate slowly. A few days later prismatic crystals were obtained.

\section{II.2.Single crystal structure determination}

The X-ray data collection was carried out on Enraf-Nonius Kappa CCD diffractometer using Ag Ka radiation .The positional parameters for the heavy atoms were obtained from a three-dimensional Patterson map, while the non- $\mathrm{H}$ atoms were found from successive difference Fourier Maps. The structure was refined by full-matrix least squares using anisotropic temperature factors for all non-hydrogen atoms and the hydrogen atoms were localized and optimized to restrained positions. Calculations were performed with the programs SHELXS [21], using the scattering factors enclosed therein. The crystal data, collected reflections and parameters of the final refinement are reported in Table 1.

Table 1. Crystal and experimental data

\begin{tabular}{ll}
\hline Chemical formula & {$\left[\mathrm{C}_{7} \mathrm{H}_{22} \mathrm{~N}_{3}\right] \mathrm{BiCl}_{6}$} \\
Formula weight & $440.37(6)$ \\
Crystal system & Triclinic \\
Space group & $P 1$ \\
$T$ & $293(2) \mathrm{K}$ \\
Cell constants & \\
$a$ & $7,5580(5) \AA$ \\
$b$ & $7,8710(6) \AA$ \\
$c$ & $17.3168(13) \AA$ \\
$\beta$ & $89,169(5)^{\circ}$ \\
Cell volume $\left(\AA^{3}\right)$ & $3005.5(4)$ \\
$Z$ & 1 \\
Dx & $2,15 \mathrm{~g} / \mathrm{cm}^{3}$ \\
Diffractometer/scan & Enraf-Nonius Kappa CCD \\
\hline
\end{tabular}




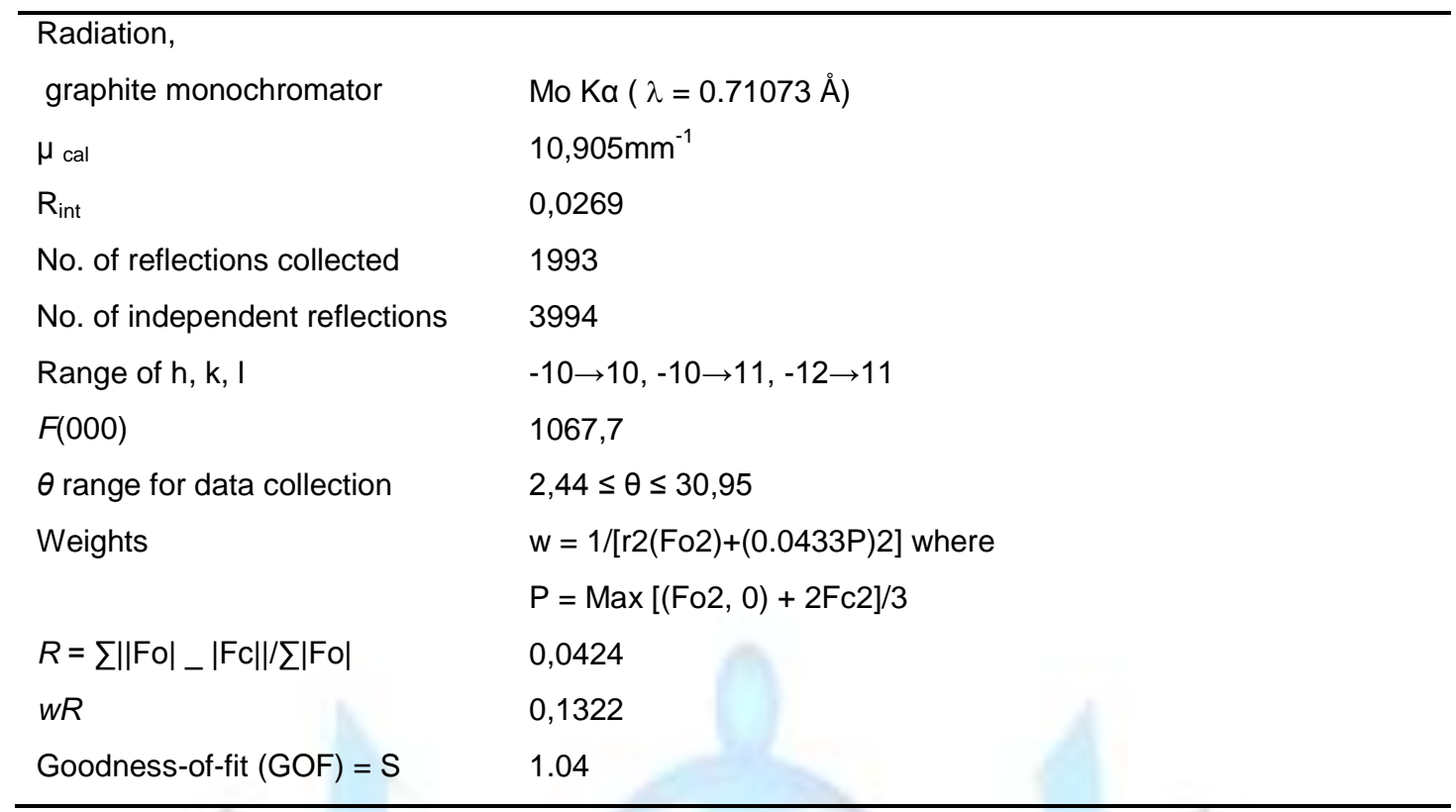

\section{Results and discussion}

The final atomic coordinates obtained from the single crystal refinement with Ueq are given in Table 2. Table 3 shows the anisotropic displacement parameters. Interatomic distances and bond angles schemes are listed in Table 4.

The title compound crystallizes in the non-centrosymmetric triclinic space group P1. The crystallographic analysis of the title compound reveals that the crystal structure of $\left[\mathrm{C}_{7} \mathrm{H}_{22} \mathrm{~N}_{3}\right] \mathrm{BiCl}$ consists of the monomeric $\left[\mathrm{BiCl}_{6}\right]^{3-}$ anion and one crystallographically independent triprotonated 3-3'-diamino- $\mathrm{N}$-methyldipropylammonium cation, as shown in Figure 1.

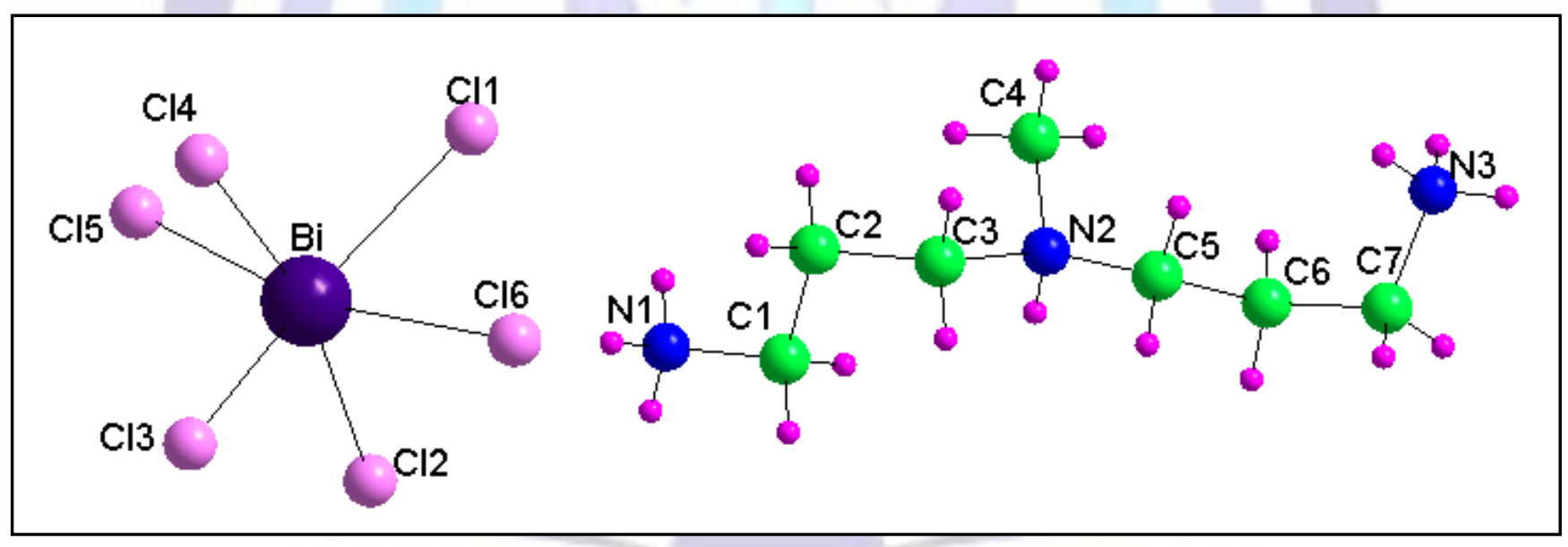

Fig 1: Atom numbering scheme for the title compound $\left[\mathrm{C}_{7} \mathrm{H}_{22} \mathrm{~N}_{3}\right] \mathrm{BiCl}_{6}$ 
Table 2 . Atomic coordinates and $U_{\text {eq }}$ or $U_{\text {iso }}$ for $\left[\mathrm{C}_{7} \mathrm{H}_{22} \mathrm{~N}_{3}\right] \mathrm{BiCl}_{6}$

\begin{tabular}{|c|c|c|c|c|}
\hline Atomes & $X / a$ & $Y / b$ & $Z / c$ & $U_{\text {éq }}$ ou $U_{\text {iso }}$ \\
\hline $\mathrm{Bi}$ & $-0,0877$ & $-0,0867$ & $-0,0788$ & $0,02492(11)$ \\
\hline $\mathrm{Cl} 1$ & $-0,4010(5)$ & $0,0629(5)$ & $0,1204(4)$ & $0,0407(7)$ \\
\hline $\mathrm{Cl} 2$ & $-0,2031(9)$ & $0,2085(8)$ & $-0,3160(6)$ & $0,0553(12)$ \\
\hline $\mathrm{Cl} 3$ & $0,3009(5)$ & $-0,2504(7)$ & $-0,1459(5)$ & $0,0477(8)$ \\
\hline $\mathrm{Cl} 4$ & $-0,2566(6)$ & $-0,2675(5)$ & $-0,2373(4)$ & $0,0368(6)$ \\
\hline $\mathrm{Cl} 5$ & 0,0978 (8) & $-0,4189(7)$ & $0,1099(7)$ & $0,0521(11)$ \\
\hline $\mathrm{Cl} 6$ & $-0,0346(8)$ & $0,1401(8)$ & $0,1127(8)$ & $0,0717(17)$ \\
\hline $\mathrm{C} 1$ & $-0,442(2)$ & $-0,372(2)$ & $0,1143(17)$ & $0,038(3)$ \\
\hline $\mathrm{H} 1 \mathrm{~A}$ & $-0,5607$ & $-0,2488$ & 0,0954 & $0,046^{*}$ \\
\hline $\mathrm{H} 1 \mathrm{~B}$ & $-0,3270$ & $-0,3506$ & 0,0929 & $0,046^{*}$ \\
\hline C2 & $-0,432(3)$ & $-0,452(3)$ & $0,2918(18)$ & $0,040(3)$ \\
\hline $\mathrm{H} 2 \mathrm{~A}$ & $-0,5449$ & $-0,4777$ & 0,3125 & $0,049^{*}$ \\
\hline $\mathrm{H} 2 \mathrm{~B}$ & $-0,3111$ & $-0,5720$ & 0,3123 & $0,049^{*}$ \\
\hline C3 & $-0,436(2)$ & $-0,311(3)$ & $0,4006(19)$ & $0,041(3)$ \\
\hline $\mathrm{H} 3 \mathrm{~A}$ & $-0,3285$ & $-0,2798$ & 0,3717 & $0,049^{*}$ \\
\hline $\mathrm{H} 3 \mathrm{~B}$ & $-0,4066$ & $-0,3733$ & 0,5092 & $0,049^{\star}$ \\
\hline C4 & $-0,802(3)$ & $-0,161(3)$ & $0,435(2)$ & $0,045(3)$ \\
\hline $\mathrm{H} 4 \mathrm{~A}$ & $-0,8031$ & $-0,2459$ & 0,3600 & $0,068^{*}$ \\
\hline $\mathrm{H} 4 \mathrm{~B}$ & $-0,9243$ & $-0,0431$ & 0,4227 & $0,068^{*}$ \\
\hline $\mathrm{H} 4 \mathrm{C}$ & $-0,7885$ & $-0,2202$ & 0,5421 & $0,068^{*}$ \\
\hline C5 & $-0,605(3)$ & $0,007(2)$ & $0,499(2)$ & $0,044(4)^{*}$ \\
\hline $\mathrm{H} 5 \mathrm{~A}$ & $-0,4764$ & 0,0024 & 0,4774 & $0,053^{*}$ \\
\hline $\mathrm{H} 5 \mathrm{~B}$ & $-0,6018$ & $-0,0396$ & 0,6112 & $0,053^{*}$ \\
\hline C6 & $-0,772(3)$ & $0,224(2)$ & $0,473(2)$ & $0,043(4)$ \\
\hline $\mathrm{H} 6 \mathrm{~A}$ & $-0,7593$ & 0,2770 & 0,3669 & $0,051^{*}$ \\
\hline $\mathrm{H} 6 \mathrm{~B}$ & $-0,9014$ & 0,2262 & 0,4758 & $0,051^{*}$ \\
\hline C7 & $-0,769(3)$ & $0,352(3)$ & $0,590(3)$ & $0,052(4)$ \\
\hline $\mathrm{H} 7 \mathrm{~A}$ & $-0,6339$ & 0,3316 & 0,6047 & $0,062^{*}$ \\
\hline H7B & $-0,8501$ & 0,4842 & 0,5475 & $0,062^{*}$ \\
\hline N1 & $-0,447(2)$ & $-0,5025(18)$ & $0,0071(15)$ & $0,039(2)$ \\
\hline HN1A & $-0,4528$ & $-0,4538$ & $-0,0940$ & $0,058^{\star}$ \\
\hline HN1B & $-0,5545$ & $-0,5199$ & 0,0254 & $0,058^{*}$ \\
\hline $\mathrm{HN1C}$ & $-0,3382$ & $-0,6146$ & 0,0235 & $0,058^{*}$ \\
\hline N2 & $-0,632(2)$ & $-0,1186(18)$ & $0,4022(15)$ & $0,037(3)$ \\
\hline HN2 & $-0,6525$ & $-0,0610$ & 0,3001 & $0,045^{\star}$ \\
\hline N3 & $-0,844(2)$ & $0,319(2)$ & $0,744(2)$ & $0,057(4)$ \\
\hline HN3A & $-0,8398$ & 0,3993 & 0,8100 & $0,085^{\star}$ \\
\hline HN3B & $-0,7680$ & 0,1984 & 0,7843 & $0,085^{\star}$ \\
\hline HN3C & $-0,9686$ & 0,3390 & 0,7309 & $0,085^{\star}$ \\
\hline
\end{tabular}


The atomic arrangement of the complex is depicted in Figure 2. An examination shows a layer arrangement paralleled to the $\mathrm{b}$ direction: octahedral of $\left[\mathrm{BiCl}_{6}\right]^{3-}$ alternate with planes of 3-3'-diamino-N-méthyldipropylammonium groups. The monomeric $\left[\mathrm{BiCl}_{6}\right]^{3-}$ anions are located in the $(\mathrm{b}, \mathrm{c})$ plane at $\mathrm{z}=0$ and $z=1$ and those for organic groups at $z=1 / 2$.

Table 3. Anisotropic displacemant parametrs $\left(\AA^{2}\right)$ for $\left[\mathrm{C}_{7} \mathrm{H}_{22} \mathrm{~N}_{3}\right] \mathrm{BiCl}_{6}$

\begin{tabular}{lllllll}
\hline Atomes & U11 & U22 & U33 & U23 & U13 & U12 \\
\hline Bi & $0,02398(14)$ & $0,02008(15)$ & $0,02926(16)$ & $-0,00296(9)$ & $-0,00009(10)$ & $-0,00875(10)$ \\
Cl1 & $0,0389(15)$ & $0,0403(17)$ & $0,0446(17)$ & $-0,0110(13)$ & $0,0116(13)$ & $-0,0188(13)$ \\
Cl2 & $0,061(3)$ & $0,048(3)$ & $0,053(2)$ & $0,022(2)$ & $-0,012(2)$ & $-0,027(2)$ \\
Cl3 & $0,0345(14)$ & $0,062(2)$ & $0,0412(17)$ & $0,0043(15)$ & $0,0002(13)$ & $-0,0196(16)$ \\
Cl4 & $0,0413(16)$ & $0,0361(16)$ & $0,0370(15)$ & $-0,0036(12)$ & $-0,0037(12)$ & $-0,0210(14)$ \\
Cl5 & $0,055(2)$ & $0,042(2)$ & $0,063(3)$ & $0,011(2)$ & $-0,014(2)$ & $-0,027(2)$ \\
Cl6 & $0,052(2)$ & $0,056(3)$ & $0,092(4)$ & $-0,043(3)$ & $-0,023(3)$ & $-0,004(2)$ \\
N2 & $0,050(7)$ & $0,025(5)$ & $0,028(5)$ & $-0,010(4)$ & $0,003(5)$ & $-0,009(5)$ \\
C3 & $0,036(6)$ & $0,041(9)$ & $0,035(7)$ & $0,012(6)$ & $-0,013(5)$ & $-0,012(6)$ \\
C5 & $0,056(9)$ & $0,040(8)$ & $0,046(8)$ & $0,016(7)$ & $-0,028(7)$ & $-0,032(8)$ \\
C4 & $0,056(9)$ & $0,051(9)$ & $0,045(7)$ & $-0,013(6)$ & $0,011(7)$ & $-0,037(8)$ \\
C2 & $0,054(9)$ & $0,032(8)$ & $0,030(6)$ & $0,000(5)$ & $-0,008(6)$ & $-0,016(7)$ \\
C6 & $0,045(8)$ & $0,019(6)$ & $0,056(10)$ & $0,002(6)$ & $0,000(7)$ & $-0,009(6)$ \\
C1 & $0,049(7)$ & $0,031(6)$ & $0,040(6)$ & $-0,004(5)$ & $0,004(6)$ & $-0,023(6)$ \\
C7 & $0,051(9)$ & $0,041(10)$ & $0,064(11)$ & $-0,016(8)$ & $-0,003(8)$ & $-0,019(8)$ \\
N1 & $0,041(6)$ & $0,032(6)$ & $0,038(6)$ & $-0,011(4)$ & $0,000(5)$ & $-0,012(5)$ \\
N3 & $0,043(7)$ & $0,049(8)$ & $0,060(9)$ & $-0,025(7)$ & $-0,004(6)$ & $-0,002(6)$ \\
\hline
\end{tabular}

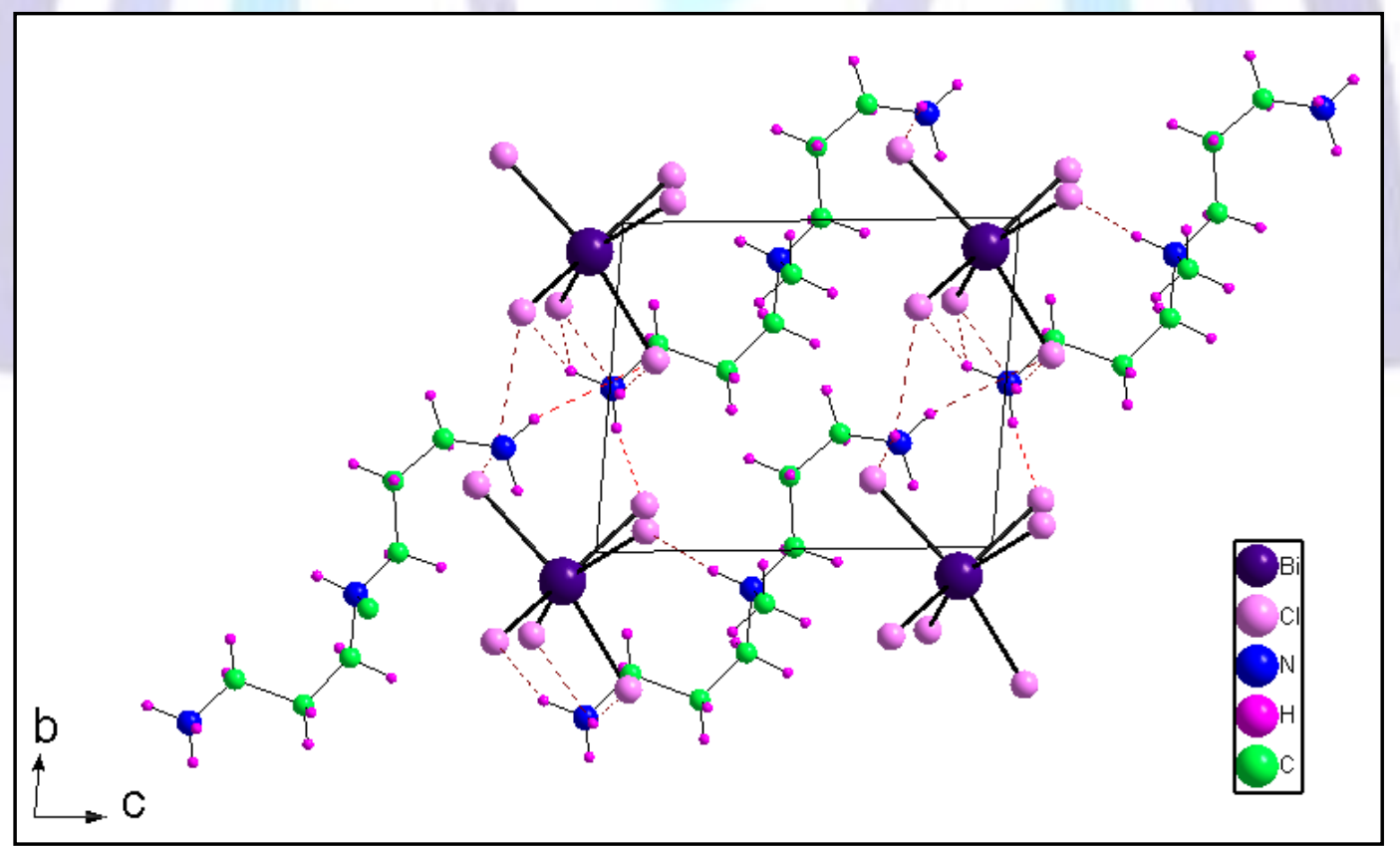

Fig 2: Projection in the plane (b,c) of the atomic arrangement of $\left[\mathrm{C}_{7} \mathrm{H}_{22} \mathrm{~N}_{3}\right] \mathrm{BiCl}_{6}$

Each $\mathrm{Bi}$ atom is surrounded by six $\mathrm{Cl}$ atoms, forming a distorted octahedral configuration, with $\mathrm{Bi}-\mathrm{Cl}$ bond lengths ranging from $2,668(4)$ to $2,734(3) \AA$ (Table 4 ) and a mean value of $2.727(2) \AA$, and $\mathrm{Cl}-\mathrm{Bi}-\mathrm{Cl}$ bond angles ranging from $71.87(2)$ to $97.91(3)^{\circ}$ for cis and from $100.21(2)$ to $166.56(2)^{\circ}$ for trans arrangements. These values are comparable with those reported to date [20]. 
In addition to the bond length differences, the $\mathrm{Cl}-\mathrm{Bi}-\mathrm{Cl}$ bond angles (Table 2) deviate from $90^{\circ}$, with the biggest difference $\left[97.91(3)^{\circ}\right]$ occurring for the $\mathrm{Cl}(6)-\mathrm{Bi}-\mathrm{Cl}(5)$ angle and the lowest one $\left[71.87(2)^{\circ}\right]$ occurring for the $\mathrm{Cl}(3)-\mathrm{Bi}-\mathrm{Cl}(5)$ angle, which incorporates the two shortest $\mathrm{Bi}-\mathrm{Cl}$ bonds. This distortion is correlated both to primary deformations resulting from the stereochemical activity of the Bi lone electron pair $[20,22]$ and to secondary deformations resulting from hydrogen bond interactions [23].

The involvement of any particular chlorine atom in hydrogen bonding brings about the shift of the lone electron pair of the $\mathrm{Bi}$ atom in the direction of the $\mathrm{H}$ atom, which generally results in the shift of the respective $\mathrm{Cl}$ atom out of the $\mathrm{Bi}$ position. This leads to an increase in the $\mathrm{Bi}-\mathrm{Cl}$ bond length (Bi-Cl1: 2.73(3), $\mathrm{Bi}-\mathrm{Cl} 5: 2.730(4)$ and $\mathrm{Bi}-\mathrm{Cl} 4: 2.734(3) \AA$ ) compared to the others.

Figure 2 shows that the 3-3'-diamino- $\mathrm{N}$-methyldipropylammonium cations are interspersed between the anionic plans at $z=1 / 2$. The $\mathrm{C}-\mathrm{N}$ and $\mathrm{C}-\mathrm{C}$ bond lengths vary respectively from $1.442(2)$ to $1.56(2)$ and $1.47(3)$ to $1.59(2) \AA$. These values are comparable with those reported by other researchers [24].

Table4. Principal intramolecular distances $(\AA)$, bond angles $\left(^{\circ}\right)$ in $\left[\mathrm{C}_{7} \mathrm{H}_{22} \mathrm{~N}_{3}\right] \mathrm{BiCl}_{6}$

\begin{tabular}{|c|c|c|c|}
\hline Atoms & Distance & Atoms & Distance \\
\hline $\mathrm{Bi}-\mathrm{Cl} 1$ & $2,730(3)$ & C3- C2 & 1,49 (3) \\
\hline $\mathrm{Bi}-\mathrm{Cl} 2$ & $2,730(4)$ & C5- C6 & $1,59(2)$ \\
\hline $\mathrm{Bi}-\mathrm{Cl} 3$ & $2,686(4)$ & $\mathrm{C} 2-\mathrm{C} 1$ & $1,550(2)$ \\
\hline $\mathrm{Bi}-\mathrm{Cl} 4$ & $2,734(3)$ & $\mathrm{C} 6-\mathrm{C} 7$ & 1,47 (3) \\
\hline $\mathrm{Bi}-\mathrm{Cl} 5$ & $2,692(5)$ & $\mathrm{C} 1-\mathrm{N} 1$ & $1,440(2)$ \\
\hline \multirow[t]{5}{*}{$\mathrm{Bi}-\mathrm{Cl} 6$} & $2,668(4)$ & $\mathrm{C} 7-\mathrm{N} 3$ & 1,44 (3) \\
\hline & & N2 - C5 & $1,42(2)$ \\
\hline & & N2 - C4 & $1,48(2)$ \\
\hline & & $\mathrm{N} 2-\mathrm{C} 3$ & $1,56(2)$ \\
\hline & & $\mathrm{N} 2-\mathrm{C} 3$ & $1,56(2)$ \\
\hline $\mathrm{Cl} 1-\mathrm{Bi}-\mathrm{Cl} 4$ & $92,70(2)$ & C5 - N2 -C4 & $118,3(2)$ \\
\hline $\mathrm{Cl} 2-\mathrm{Bi}-\mathrm{Cl} 1$ & $100,21(2)$ & $\mathrm{C} 5-\mathrm{N} 2-\mathrm{C} 3$ & $110,3(3)$ \\
\hline $\mathrm{Cl} 2-\mathrm{Bi}-\mathrm{Cl} 4$ & $92,46(2)$ & $\mathrm{C} 4-\mathrm{N} 2-\mathrm{C} 3$ & $109,5(3)$ \\
\hline $\mathrm{Cl} 3-\mathrm{Bi}-\mathrm{Cl} 2$ & $95,91(4)$ & $\mathrm{C} 2-\mathrm{C} 3-\mathrm{N} 2$ & $117,3(2)$ \\
\hline $\mathrm{Cl} 3-\mathrm{Bi}-\mathrm{Cl} 1$ & $153,90(3)$ & $\mathrm{N} 2-\mathrm{C} 5-\mathrm{C} 6$ & $114,4(2)$ \\
\hline $\mathrm{Cl} 5-\mathrm{Bi}-\mathrm{Cl} 2$ & $166,56(2)$ & $\mathrm{C} 3-\mathrm{C} 2-\mathrm{C} 1$ & $110,0(1)$ \\
\hline $\mathrm{Cl} 3-\mathrm{Bi}-\mathrm{Cl} 5$ & $71,87(2)$ & $\mathrm{C} 7-\mathrm{C} 6-\mathrm{C} 5$ & $116,0(2)$ \\
\hline $\mathrm{Cl} 3-\mathrm{Bi}-\mathrm{Cl} 4$ & $107,04(3)$ & $\mathrm{N} 1-\mathrm{C} 1-\mathrm{C} 2$ & $110,9(2)$ \\
\hline $\mathrm{Cl} 5-\mathrm{Bi}-\mathrm{Cl} 1$ & $93,20(2)$ & N3- C7 -C6 & $112,5(1)$ \\
\hline $\mathrm{Cl} 5-\mathrm{Bi}-\mathrm{Cl} 4$ & $86,04(3)$ & & \\
\hline $\mathrm{Cl} 6-\mathrm{Bi}-\mathrm{Cl} 1$ & $69,95(4)$ & & \\
\hline $\mathrm{Cl} 6-\mathrm{Bi}-\mathrm{Cl} 2$ & $87,6(2)$ & & \\
\hline $\mathrm{Cl} 6-\mathrm{Bi}-\mathrm{Cl} 3$ & $90,52(2)$ & & \\
\hline $\mathrm{Cl} 6-\mathrm{Bi}-\mathrm{Cl} 4$ & $162,33(2)$ & & \\
\hline $\mathrm{Cl} 6-\mathrm{Bi}-\mathrm{Cl} 5$ & $97,91(3)$ & & \\
\hline
\end{tabular}

In $\left[\mathrm{C}_{7} \mathrm{H}_{22} \mathrm{~N}_{3}\right] \mathrm{BiCl}_{6}$, the organic species interact with the inorganic chains via $\mathrm{N}-\mathrm{H}$...Cl hydrogen bonds, forming a three dimensional network (Table 4) such that all the hydrogen atoms bonded to nitrogen atoms participate in the 
formation of these hydrogen bonds, with distances between 2.310 and $3.76 \AA$. Tow of these bonds are considered as strong, whilst the others are weak [25]. Details of the hydrogen bonding scheme are reported in Table 5.

Table 5: Main inter-atomic distances $(\AA)$ and bond angles $\left(^{\circ}\right)$ involved in the hydrogen Bonds of $\left[\mathrm{C}_{7} \mathrm{H}_{22} \mathrm{~N}_{3}\right] \mathrm{BiCl}_{6}$ compounds.

\begin{tabular}{lllll}
\hline $\mathrm{D}-\mathrm{H} \ldots \mathrm{A}$ & $\mathrm{d}(\mathrm{D}-\mathrm{H})$ & $\mathrm{d}(\mathrm{H} \ldots \mathrm{A})$ & $\mathrm{d}(\mathrm{D} \ldots \mathrm{A})$ & $\mathrm{d}(\mathrm{DHA})$ \\
\hline $\mathrm{N}_{2}-\mathrm{H}_{2} \ldots \mathrm{Cl}_{1}$ & 0,910 & 2,860 & 127,85 & 3,493 \\
$\mathrm{~N}_{2}-\mathrm{H}_{2} \ldots \mathrm{Cl}_{6}{ }^{\mathrm{a}}$ & 0,910 & 2,964 & 127,63 & 3,593 \\
$\mathrm{~N} 1-\mathrm{H}_{1 \mathrm{a}} \ldots \mathrm{Cl}_{3}{ }^{\mathrm{a}}$ & 0,890 & 1,838 & 110,91 & 3,310 \\
$\mathrm{~N}_{1}-\mathrm{H}_{1 \mathrm{a}} \ldots \mathrm{Cl}_{4}$ & 0,890 & 2,722 & 130,64 & 3,370 \\
$\mathrm{~N}_{1}-\mathrm{H}_{1 \mathrm{~b}} \ldots \mathrm{Cl}_{5}{ }^{\mathrm{a}}$ & 0,890 & 2,484 & 153,88 & 3,306 \\
$\mathrm{~N}_{1}-\mathrm{H}_{1 \mathrm{c}} \ldots \mathrm{Cl}_{6}{ }^{\mathrm{b}}$ & 0,890 & 2,296 & 164,60 & 3,163 \\
$\mathrm{~N}_{1}-\mathrm{H}_{1 \mathrm{c}} \ldots \mathrm{Cl}_{1}{ }^{\mathrm{b}}$ & 0,890 & 2,837 & 115,88 & 3,323 \\
$\mathrm{~N}_{3}-\mathrm{H}_{3 \mathrm{a}} \ldots \mathrm{Cl}_{5}{ }^{\mathrm{c}}$ & 0,890 & 2,928 & 156,29 & 3,760 \\
$\mathrm{~N}_{3}-\mathrm{H}_{3 \mathrm{c}} \ldots \mathrm{Cl}_{2}{ }^{\mathrm{d}}$ & 0,890 & 2,473 & 148,90 & 3,268 \\
$\mathrm{~N}_{3}-\mathrm{H}_{3 \mathrm{c}} \ldots \mathrm{Cl}_{4}{ }^{\mathrm{c}}$ & 0,890 & 2,889 & 112,63 & 3,334 \\
\hline
\end{tabular}

Symmetry codes:

$a:[x-1, y, z], \quad b:[x, y-1, z] \quad, \quad c:[x-1, y+1, z+1], \quad d:[x-1, y, z+1]$

\section{Conclusion}

The 3-3'-diamino-N-methyldipropylammonium hexachlorobismuthate (III) belongs to the triclinic system with P1 space group. The crystal structure consists of the monomeric $\left[\mathrm{BiCl}_{6}\right]^{3-}$ and one crystallographically independent triprotonated 3-3'-diamino-N-methyldipropylammonium cation. The structure of this compound consists of alternation organic and inorganic layers. These layers are themselves interconnected by means of the ionic $\mathrm{N}-\mathrm{H}$... Cl hydrogen bonds.

\section{ACKNOWLEDGEMENTS}

This work is supported by the Tunisian National Ministry of Higher Education and Scientific Research.

\section{REFERENCES}

1. Mitzi, D.B, Chondroudis, O. Kagan, C, Inorg. Chem. 38 (1999) 6246-6256.

2. Papavassiliou, G.C, Solid State Chem. 40 (1981) 330-335.

3. Era, M. Morimoto, S. Tsutsui, T. Saito, S, Appl. Phys. Lett. 65 (1994) 676.

4. Wojtas, M. Jakubas, R. Ciunik, Z. Medycki, W, J. Solid State Chem. 177 (2004) 1575-1584.

5. Bator, G. Zeegers-Huyskens, Th. Jakubas, R. Zaleski, J, J. Mol. Struct. 570 (2001) 61 -74.

6. Kulicka, B. Lis, T. Kinzhybalo, V. Jakubas, R. Piecha, A. Polyhedron 29 (2010) 2014 -2022.

7. Zhu, X-H. Mercier, N. Fre're, P. Blanchard, P. Roncali, J. Allain,M. Pasquier, C. Riou,A . Inorg Chem 42(2003) 5330-5339.

8. Ryan, JM. Xu, Z. Inorg Chem 43 (2004) 4106-4108.

9. Mitzi, DB. Inorg Chem 39 (2000) 6107-6113.

10. Xu, Z. Mitzi, DB. Inorg Chem 42(2003) 6589-6591.

11. Carmalt, CJ. Farrugia, LJ. Norman, NC. Z Anorg Allg Chem 621 (1995) 47-56.

12. Carmalt, CJ. Farrugia, LJ. Norman, NC. Z Naturforsch Teil B 50(1995) 1591-1596.

13. Ryan, JM. Xu, Z. Inorg Chem 40(2001) 2096-2104.

14. Lefebvre, J. Carpentier, P. Jakubas, R. Acta Crystallogr B47 (1991) 228-234. 
15. Ishihara, S. Dou, A. Weiss Ber Bunsenges. Phys Chem 95(1991) 659-670.

16. Blachnik, R. Jaschinski, B. Reuter, H. Kauster, GZ. Kristallografia 212(1997) 874-879.

17. Fisher, GA. Norman, NC. Adv Inorg Chem. 41(1994) 233-271.

18. Chaabouni, S. Kammoun, S. Jaud, J. J Chem. Crystallogr 27(1997) 527-531.

19. Hrizi, C. Chaabouni, S. Jpn Soc Anal Chem 26 (2010) 3-7.

20. Chaabouni, S. Kamoun, S. Jaud, J. J. Chem. Crystallogr. 28 (1998) 209-212.

21. Sheldrick, G.M. Acta Crystallogr Sect. A 64 (2008) 112-122.

22. Wang, X. Liebau, F. Acta Crystallogr Sect. B 52 (1996) 1-6.

23. Zaleski, J. Pietraszko, A. Acta Crystallogr Sect. B 52 (1996) 287-295.

24. De Almeida, B. Back, D. Lang, E. Felcman. J Inorg Chim Acta 362 (2009) 2447-2451.

25. Pimental, G.C. Mc Clellan, A.L, the Hydrogen Bond, Freeman, San Fransisco, 1971. 